\title{
A comparison of lysis centrifugation, pour plate, and conventional blood culture methods in the diagnosis of septicaemic melioidosis
}

\author{
A J H Simpson, P A Howe, V Wuthiekanun, N J White
}

\begin{abstract}
Aims-To determine whether quantitative blood culture methods could improve the diagnosis of septicaemic melioidosis.

Methods-A comparison of conventional broth based blood cultures, a pour plate method, and a commercial lysis centrifugation (Isolator $10^{\mathrm{TM}}$ ) blood culture system was conducted in 71 Thai patients with severe melioidosis. The time to identification of $B$ pseudomallei was recorded for each method.

Results-42 patients (59\%) were septicaemic. Compared with conventional blood culture, the Isolator and pour plate methods had sensitivities of $81 \%$ and $61 \%$, respectively. The median times to a positive culture were: Isolator 39.3 hours, pour plates 45.5 hours, broth culture 61.8 hours ( $p<0.001$ Isolator $v$ broth). There was a significant inverse correlation between Isolator tube or pour plate quantitative counts and time to detection $(r=-0.44$ and -0.57 , respectively). Mortality was higher in patients who were septicaemic. Conclusions-Routine use of one of these quantitative methods, in addition to conventional broth culture, may lead to earlier diagnosis of septicaemic melioidosis.

(f Clin Pathol 1999;52:616-619)
\end{abstract}

Keywords: melioidosis; pour plate; lysis centrifugation; blood culture methods

Faculty of Tropical

Medicine, Mahidol

University, $420 / 6$

Rajvithi Road,

Bangkok 10400,

Thailand

V Wuthiekanun

Centre for Tropical Medicine, Nuffield

Department of Clinical

Medicine, John

Radcliffe Hospital,

University of Oxford,

Oxford OX3 9DU, UK

A J H Simpson

P A Howe

N J White

Correspondence to:

Dr Andrew Simpson, Faculty

of Tropical Medicine,

Mahidol University, 420/6

Rajvithi Road, Bangkok

10400, Thailand.

email: fnajs@diamond.

mahidol.ac.th

Accepted for publication 14 April 1999 or rapid identification of the causative organism. Although a rapid presumptive diagnosis can be made using a specific immunofluorescence (IF) test, ${ }^{5}$ this relies on the availability of suitable specimens (such as sputum, urine, or pus) and an immunofluorescence microscope. Approximately $60 \%$ of patients with melioidosis are septicaemic on admission to hospital. ${ }^{3}$ Blood cultures may become positive within 24 hours, but subculture and identification of the organism requires further time. More rapid methods for diagnosis and confirmation of septicaemic melioidosis would facilitate earlier appropriate antibiotic treatment.

We report a study comparing speed of isolation of $B$ pseudomallei from conventional broth based blood cultures, a pour plate method, and the Wampole Isolator $10^{\mathrm{TM}}$ lysis centrifugation blood culture system, in adult Thai patients with severe melioidosis.

\section{Methods}

Adult patients (aged $\geqslant 15$ years) admitted to Sappasitprasong Hospital, Ubon Ratchathani, with suspected severe melioidosis were studied if they were eligible for entry into a separate open, randomised trial of ceftazidime versus imipenem treatment. ${ }^{6}$ Blood cultures (two or three $5 \mathrm{ml}$ blood samples, each inoculated into broth media) were collected at entry, together with three $\times 1 \mathrm{ml}$ sterile heparinised blood samples ${ }^{7}$ collected for quantification of bacteraemia. Cultures of sputum, throat swabs, urine, and pus (from wounds, other skin lesions, or aspirated from visceral abscesses) were performed. Blood culture bottles (TSC, Heywood, Lancashire, UK) contained $45 \mathrm{ml}$ brainheart infusion (BHI) broth with sodium polyanethol sulphonate (SPS). For this study, an additional $10 \mathrm{ml}$ of blood was inoculated aseptically into a pre-evacuated Isolator 10 lysis centrifugation blood culture tube (Unipath, Basingstoke, Hampshire, UK). The Isolator 10 tubes contain SPS, saponin, and polypropylene glycol in aqueous solution.

Broth blood culture bottles were vented (using BCB Vent/Sub units; Difco, West Molesey, Surrey, UK) and incubated unshaken in air for seven days at $37^{\circ} \mathrm{C}$. The bottles were inspected daily and Gram stains performed on smears prepared at 12-24 hours and 36-48 hours, or when visible growth occurred. Bottles were subcultured routinely onto horse blood agar at 12-24 and 36-48 hours and after seven days of incubation, and were subcultured on days 3-6 if there was any suggestion of visible growth (that is, cloudy broths). 
Table 1 Comparison of times to first positive culture with different blood culture methods

\begin{tabular}{lllll}
\hline Method & $\begin{array}{l}\text { Number } \\
\text { positive }\end{array}$ & $\begin{array}{l}\text { Median time to } \\
\text { positive }(h)\end{array}$ & Range $(h)$ & $\begin{array}{l}\text { Mean }(S D) \text { time to } \\
\text { positive }(h)\end{array}$ \\
\hline BHI broth & 42 & 61.8 & 35 to 205.5 & $78.9(51.2)$ \\
Isolator $^{\mathrm{TM}}$ & 34 & 39.3 & 16 to 64.5 & $37.2(11.7)$ \\
Pour plate & 25 & 45.5 & 16 to 91.5 & $45.5(12.8)$ \\
\hline
\end{tabular}

BHI, brain-heart infusion.

Pour plates were prepared by mixing $1 \mathrm{ml}$ of heparinised blood with $19 \mathrm{ml}$ of molten $\left(50^{\circ} \mathrm{C}\right)$ sterile Oxoid Columbia agar (Unipath) in a sterile Petri dish, as previously described. ${ }^{7}$

Isolator tubes were inverted at least 10 times after inoculation to ensure adequate mixing. They were transported immediately to the microbiology laboratory and after a minimum of 10 minutes post-inoculation, the tubes were centrifuged at $1500 \mathrm{~g}$ and $4^{\circ} \mathrm{C}$ for 30 minutes (unbraked). The supernatant (approximately 9 $\mathrm{ml}$ ) was removed aseptically to leave $1 \mathrm{ml}$ fluid plus sediment. The sediment was homogenised using a sterile pipette, and $0.5 \mathrm{ml}$ volumes were inoculated onto both horse blood and Ashdown's selective agar plates. ${ }^{8}$

All agar plates were incubated for four days at $37^{\circ} \mathrm{C}$ in air and examined daily at a set time. Colony counts were performed on relevant plates to allow calculation of the number of colony forming units per millilitre $(\mathrm{cfu} / \mathrm{ml})$ of blood.

Statistical analyses were performed using the statistical computing package SPSS for Windows 7.5 (SPSS Inc, Chicago, Illinois, USA).

\section{Results}

Between August 1996 and November 1997, 82 patients were enrolled into the study. Of these, $71(86.6 \%)$ were proved subsequently to have melioidosis (that is, cultures were positive for B pseudomallei) and 42 patients (59.2\%) had positive blood cultures. Three broth culture bottles were inoculated in 35 of the 42 septicaemic patients. Of these, 21 cultures $(60 \%)$ were positive in all three bottles, while eight $(23 \%)$ and six $(17 \%)$ were positive in only one or two bottles, respectively. Two bottles were collected in six patients; five were positive in both bottles. In the final patient only one bottle was collected. Thus all bottles were positive in 27 patients (63\%). Gram negative rods were seen in stained routine smears from blood culture broths in only four patients $(9.5 \%)$.

$B$ pseudomallei was isolated from the Isolator tube (limit of detection $0.1 \mathrm{cfu} / \mathrm{ml}$ ) in 34

$(47.9 \%)$ of the 71 patients, while $25(35.3 \%)$ of

Table 2 Quantitative bacterial counts in peripheral blood by Isolator ${ }^{T M}$ or pour plate methods and related mortality (all blood culture positive patients, $n=42$ )

\begin{tabular}{lllllll}
\hline \multicolumn{5}{l}{ Count (cfu/ml) } \\
\cline { 2 - 6 } & 0 & $<1$ & $1-10$ & $11-40$ & $41-100^{*}$ & $>100$ \\
\hline $\begin{array}{l}\text { Pour plates } \\
\text { n (\%) }\end{array}$ & $16(39.0)$ & 0 & $10(24.4)$ & $3(7.3)$ & $7(17.1)$ & $5(12.2)$ \\
$\quad$ Mortality (\%) & $7(43.8)$ & - & $5(50.0)$ & $3(100)$ & $6(85.7)$ & $5(100)$ \\
Isolator & & & & & & \\
$\quad$ n (\%) & $8(19.0)$ & $6(14.3)$ & $16(38.1)$ & $5(11.9)$ & $7(16.7)$ & \\
$\quad$ Mortality (\%) & $3(37.5)$ & $4(66.7)$ & $8(50.0)$ & $4(80.0)$ & $7(100)$ & \\
\hline
\end{tabular}

cfu, colony forming unit.

${ }^{\star}$ Count $>40 \mathrm{cfu} / \mathrm{ml}$ for Isolator.
69 patients had positive pour plates (limit of detection $0.3 \mathrm{cfu} / \mathrm{ml}$ ). Two patients did not have pour plate cultures performed. All patients with either positive pour plates or Isolators were also blood culture positive by the standard broth method. Thus $81.0 \%$ of bacteraemic patients (34 of 42) were also positive using the Isolator tube and $61.0 \%$ (25 of 42 ) had positive pour plates. All the septicaemic patients $(n=6)$ in whom only two broth culture bottles were collected (that is, $10 \mathrm{ml}$ of blood were cultured) were also Isolator positive $(100 \%)$. Eight patients were Isolator tube positive when pour plate cultures were negative (that is, they had counts of $0.1-0.3 \mathrm{cfu} / \mathrm{ml}$ ); thus the sensitivity of pour plates compared with the Isolator was $75.8 \%$. The pour plates from one patient with $B$ pseudomallei cultured from the Isolator were contaminated and unreadable.

\section{TIME TO FIRST POSITIVE CULTURE}

Results for time to recognition of a positive culture for each method are shown in table 1. This was the time at which a microbiological diagnosis of melioidosis could be made by identification of $B$ pseudomallei. In the vast majority of cases this was when colonies were available on agar media for further tests such as a specific latex agglutination test. The median time to first recognition of a positive culture for the Isolator was 39.3 hours, compared with 61.8 hours for the broth culture method $(\mathrm{p}<0.001$, Wilcoxon signed rank test) and 45.5 hours for pour plates $(\mathrm{p}=0.003)$. The pour plate method was also significantly faster than BHI blood culture broths (45.5 hours $v$ 61.8 hours; $p=0.02$ ).

Colonies were available on solid media within 24 hours using the Isolator in nine cases (21.4\% of all positive blood cultures), from pour plates in one case, and from blood culture broths in none. At 48 hours the numbers were $32(76.2 \%), 22(52.4 \%)$, and $18(42.9 \%)$ cases, respectively.

Of the four patients in whose blood culture broths bacteria were seen on Gram stain, two were detected within 24 hours and a third within 48 hours. In only one case were Gram negative rods seen in broth cultures before the Isolator cultures became positive (17 $v 41$ hours).

\section{COUNTS}

Plate count details for the Isolator and the pour plate methods are shown in table 2 . Seven of the 34 positive patients by the Isolator method had very high counts, with at least $40 \mathrm{cfu} / \mathrm{ml}$ of blood (that is, $>200 \mathrm{cfu} /$ plate), but growth was too confluent to allow exact counts. The same applied to two pour plate positive patients $(>200 \mathrm{cfu} / \mathrm{ml})$. The Isolator tube method was capable of detecting bacteraemia at lower counts than the pour plate method. However, Isolator tube counts (per $\mathrm{ml}$ of blood) were significantly lower than pour plate counts from the same patients, where these could be compared $(\mathrm{p}=0.015$, Wilcoxon signed rank test; $\mathrm{n}=20$ ). There was a good positive correlation between the counts in positive cases for the two methods (Spearman rank correlation coefficient $(r)=0.89, \mathrm{p}<0.001)$, but at the 


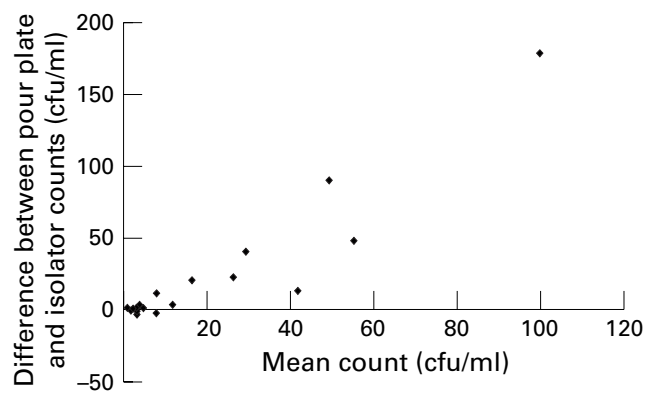

Figure 1 Discrepancies between pour plate and Isolator bacterial counts. Each point represents one patient. The figure shows the relation between the mean quantitative count for both methods combined and the difference in counts between each method, in individual patients. Pour plates generally produced higher counts; at high mean counts the differences were more pronounced.

higher counts the differences in counts were more marked. The discrepancies between the two methods are shown in fig 1 . For higher mean counts, these differences between the two methods are more apparent. Overall, the pour plate method gave counts that were a median of $2.5 \mathrm{cfu} / \mathrm{ml}$ higher than the Isolator (range -4 to $179 \mathrm{cfu} / \mathrm{ml}$, interquartile range -0.3 to 26.5 ).

There was also a positive correlation between the number of positive broth culture bottles and bacterial counts (Isolator: $r=0.590, \mathrm{p}<0.001$; pour plate: $r=0.422$, $\mathrm{p}=0.006)$.

\section{CORRELATION BETWEEN OUANTITATIVE COUNTS}

AND TIME TO DETECTION

There was a significant inverse correlation between Isolator tube quantitative counts and time to detection $(r=-0.44, \mathrm{p}=0.01)$, that is, higher counts were associated with more rapid detection times. This was also true of pour plates $(r=-0.57, \mathrm{p}=0.003)$. There were also significant inverse correlations between Isolator tube or pour plate quantitative counts and times to detection of positive blood culture broth results $(r=-0.72, \mathrm{p}<0.001$; and $r=-0.59, \mathrm{p}<0.001$, respectively).

\section{MORTALITY}

Overall 31 of the 71 patients (43.7\%) with melioidosis died. Mortality for patients with positive blood cultures was $61.9 \%$ (26 of 42 ) and for patients who were blood culture negative it was $17.2 \%$ (five of 29 ) $(\mathrm{p}<0.001$ ). Mortality for patients with $B$ pseudomallei isolated from the Isolator tube was $67.6 \%$ overall and for pour plate positive patients it was $76.0 \%$. For the Isolator there was no significant difference in mortality between positive and negative patients $(p=0.22$, Fisher's exact test), although mortality was significantly higher in those patients with Isolator counts of at least $10 \mathrm{cfu} / \mathrm{ml}(\mathrm{p}=0.015)$. Mortality appeared higher in those patients whose pour plates were positive than in those with negative plates, but this did not achieve statistical significance $\left(\mathrm{p}=0.079, \chi^{2}\right.$ test). Mortality related to peripheral blood quantitative bacterial counts for each method is shown in table 2 .

\section{Discussion}

The Isolator 10 lysis centrifugation tube has been shown in previous studies to have comparable sensitivity to conventional blood cultures for recovery of a wide range of organisms, although there have been some reports of reduced recovery of pseudomonads. ${ }^{9}{ }^{10}$ However, in terms of speed of diagnosis, lysis centrifugation has been reported to allow more rapid identification of organisms. ${ }^{11}$

There have been few studies of different blood culture systems in melioidosis, where speed in identifying the presence of $B$ pseudomallei is vital. Delay in diagnosis and thus appropriate treatment is a major factor contributing to mortality. $B$ pseudomallei grows well in most aerobic media, albeit more slowly than many bacterial pathogens. Quantitative bacterial counts in peripheral blood, using a pour plate method, have been correlated with outcome in melioidosis ${ }^{7}$ and thus provide prognostic information, although we did not report on speed of detection in this earlier study. Experience with a lysis centrifugation blood culture method in melioidosis has not been reported previously. The BacT/Alert automated blood culture system has been shown to detect positive blood culture bottles within 24 hours in $62.5 \%$ of septicaemic melioidosis cases, ${ }^{12}$ but additional incubation time (24-48 hours) was needed before colonies were available for identification. It is possible that routine use of an automated broth blood culture system in the present study may have improved detection times, but such a facility was not available, nor are automated systems often available, in those areas of rural southeast Asia where melioidosis is endemic. In a different series, standard BHI broth manual subcultures at 12-24 hours and 36-48 hours were positive in $52.3 \%$ and $80.8 \%$ of cases, respectively, ${ }^{13}$ but again further incubation time was necessary to confirm isolation of $B$ pseudomallei. Routine Gram staining of smears prepared from the blood culture broths, which might allow earlier recognition of Gram negative rods, was not reported in either of these two studies. Thus in practice it was a minimum of 36 hours before colonies were available on solid media and a microbiological diagnosis could be made. Further diagnostic delays are introduced if broth subcultures are not performed at 12-24 hours or if daily inspection and subculture of cloudy broths are not routine procedures. If a Gram stain and specific immunofluorescence test were available, as in our laboratory, a diagnosis of melioidosis could be made confidently at an earlier stage. However, direct immunofluorescence is not yet widely available and would be very costly and time consuming if routinely applied to all blood cultures growing Gram negative rods. Thus more rapid blood culture methods are still required.

The Isolator tube and pour plate methods used in this study had overall sensitivities in septicaemic melioidosis of $81 \%$ and $61 \%$, respectively, compared with conventional brain-heart infusion broth cultures. This reduced sensitivity means they could not replace 
conventional broth culture, but it must be balanced against the potential for improved speed of isolation and identification of $B$ pseudomallei. Both quantitative methods provided significantly earlier detection times (median times 39.3 and 45.5 hours, respectively) in septicaemic melioidosis. All the Isolator positive cases had been detected by 64.5 hours, very close to the median time to detection for conventional broths of 61.8 hours. Detection times were faster with higher peripheral blood bacterial counts for all three methods.

Different volumes of blood (10 $v 3 \mathrm{ml})$ were cultured in the two quantitative methods, and this probably explains the difference in sensitivity between them. As might be expected, the Isolator was more sensitive in detecting the presence of bacteraemia than pour plates. It was less sensitive overall than standard broth cultures, where 10 or $15 \mathrm{ml}$ blood were usually cultured, but as the Isolator method lacks an enrichment phase this is not surprising.

Bacterial counts for both quantitative methods correlated well with both the time to detection of positive broth cultures and the number of positive bottles. Although there was good correlation between the two quantitative methods, the pour plate method consistently gave higher quantitative counts. The reason for this is not clear, but clumping of organisms may occur during centrifugation.

Pour plates are an agar incorporation method of quantitative bacterial culture. By its very nature this method is likely to slow down the process of recovery of $B$ pseudomalle $i$, as the organism is an obligate aerobe and the characteristic colonial features seen with surface growth are lost. However, although slower than the Isolator and considerably less sensitive than either of the other two methods, the pour plates in our study provided a significantly more rapid method of isolation of $B$ pseudomalle $i$ colonies than did conventional BHI broths. This pour plate method is very simple and is less technically demanding than the Isolator. It is also much cheaper than the Isolator method (costs per blood tube being approximately US $\$ 0.10$ and $\$ 10.00$ respectively, compared with $\$ 1.60$ per conventional blood culture bottle). The considerable expense involved in routine use of Isolator tubes may put them beyond the budget of many hospitals in the rural tropics, and pour plates are an attractive alternative. Furthermore, the difference between median times to detection for these two methods, although significant, was only six hours, compared with another 16-22 hours for conventional broths (the equivalent of another working day).

We are unable to show from this study that the use of either quantitative method resulted in improved patient outcome, as all patients, by definition, were suspected of having acute severe melioidosis and were immediately started on treatment appropriate for this disease (ceftazidime or imipenem). Thus earlier microbiological confirmation of the diagnosis did not influence antibiotic treatment in the research setting. However, in an endemic area, where empirical regimens for community acquired septicaemia may not be effective against $B$ pseudomallei, more rapid microbiological diagnosis should result in an earlier switch to a more appropriate regimen.

This study shows that more rapid detection of septicaemic melioidosis is possible with the use of either a pour plate or lysis centrifugation blood culture system. The pour plate method is rapid and very cheap, but missed almost $40 \%$ of septicaemic patients. The Isolator lysis centrifugation method is also rapid, but is expensive and missed approximately $20 \%$ of patients, compared with conventional brainheart infusion broth culture of $15 \mathrm{ml}$ of blood. These quantitative methods also provide important prognostic information - they are most likely to be positive in those patients with the worst prognosis. The conventional broths are slow in comparison but must remain the gold standard method. Quantitative methods should thus augment rather than replace conventional broth culture in patients with suspected septicaemic melioidosis. We recommend the use of either of these quantitative methods alongside conventional broth blood culture. The Isolator tube is the preferred option owing to its greater sensitivity, but costs may dictate the use of the pour plate method in many areas where melioidosis is endemic. This should result in earlier appropriate treatment being offered to patients.

We are grateful to Julie Simpson for statistical advice. We thank Drs Wipada Chaowagul, Adul Rajanuwong, and the medical and nursing staff of the Department of Medicine, Sappasitprasong Hospital, Ubon Ratchathani, Thailand, for their help with this study, together with Nittaya Teerawattanasook and the staff of the Microbiology laboratory. Drs Brian Angus, Paul Newton, Yupin Suputtamongkol, and Petey Laohaburanakit helped with collection of specimens. We thank the Director of the hospital for allowing us to conduct these studies. This study was part of the Wellcome-Mahidol University, Oxford Tropical Medicine the Wellcome-Mahidol University, Oxford Tropical Medicine Research Programme, funded by the Wellcome Trust of Great Britain. The work was presented in part at the International Congress on Melioidosis, Bangkok, Thailand, in November Centre for Tropical Medicine, Nuffield Dept of Clinical MediCentre for Tropical Med
cine, Oxford University.

1 Dance DAB. Melioidosis: the tip of the iceberg? Clin Microbiol Rev 1991;4:52-60.

2 Chaowagul W, White NJ, Dance DAB, et al. Melioidosis: a major cause of community-acquired septicemia in northeastern Thailand. I Infect Dis 1989;159:890-8.

3 Suputtamongkol Y, Hall AJ, Dance DAB, et al. The epidemiology of melioidosis in Ubon Ratchatani, northeast Thailand. Int f Epidemiol 1994;23:1082-90.

4 White NJ, Dance DAB, Chaowagul W, et al. Halving of mortality of severe melioidosis by ceftazidime. Lancet 1989;ii:697-701.

5 Walsh AL, Smith MD, Wuthiekanun V, et al. Immunofluorescence microscopy for the rapid diagnosis of melioidosis. 7 Clin Pathol 1994;47:377-9.

6 Simpson AJH, Suputtamongkol Y, Smith MD, et al. A comparison of imipenem and ceftazidime as therapy for severe melioidosis. Clin Infect Dis (in press)

7 Walsh AL, Smith MD, Wuthiekanun V, et al. Prognostic significance of quantitative bacteremia in septicemic melionificance of quantitative bacteremia in sept
idosis. Clin Infect Dis 1995;21:1498-500.

8 Ashdown LR. An improved screening technique for Ashdown LR. An improved screening technique for
isolation of Pseudomonas pseudomallei from clinical speciisolation of Pseudomonas pseud
mens. Pathology 1979;11:293-7.

9 Brannon P, Kiehn TE. Large-scale comparison of the lysiscentrifugation and radiometric systems for blood culture. $\mathcal{F}$ Clin Microbiol 1985;22:951-4

10 Pohlman JK, Kirkley BA, Easley KA, et al. Controlled clinical comparison of Isolator and BACTEC 9240 Aerobic/F resin bottle for detection of bloodstream infections. 7 Clin Microbiol 1995;33:2525-9.

11 Hellinger WC, Cawley JJ, Alvarez S, et al. Clinical comparison of the Isolator and BacT/Alert aerobic blood culture systems. F Clin Microbiol 1995;33:1878-90.

12 Tiangpitayakorn C, Songsivilai S, Piyasangthong N, et al. Speed of detection of Burkholderia pseudomallei in blood cultures and its correlation with the clinical outcome. Am $\mathcal{f}$ cultures and its correlation with

13 Wuthiekanun V, Dance D, Chaowagul W, et al. Blood culture techniques for the diagnosis of melioidosis. Eur $\mathcal{F}$ Clin Microbiol Infect Dis 1990;9:654-8. 